\title{
Stargardt disease - what general ophthalmologists should know about this macular dystrophy
}

\author{
Wojciech Lubiński, Ewelina Lachowicz \\ $2^{\text {nd }}$ Chair and Clinic of Ophthalmology, Pomeranian Medical University, Szczecin, Poland
}

\begin{abstract}
On the basis of the available literature in the PubMed database, we describe the pathogenesis, diagnosis and therapeutic trials in Stargardt disease (STGD). STGD is the most commonly inherited cause of visual loss in childhood and adulthood. STGD is mostly inherited in an autosomal recessive pattern and is most commonly caused by mutations in the $A B C A 4$ gene leading to accumulation of the lipofuscin-like substance A2E, toxic to retinal pigment epithelium (RPE) and photoreceptors. Genetic analysis is necessary for a reliable diagnosis. STGD can be classified into 3 types with different prognoses based on the flash electroretinogram (ERG) and fundus autofluorescence (FAF). Spectral domain optical coherence tomography (SD-OCT), multifocal ERG (mfERG) and pattern
\end{abstract}

ERG (PERG) are useful in the early detection of changes. Adaptive optics scanning laser ophthalmoscopy (AOSLO) may be used to evaluate progression and for selection of patients for clinical trials. At present, there are clinical trials: gene replacement therapy (StarGen), subretinal injection of RPE cells, oral substitutes reducing the accumulation of A2E (Alkeus, Acucella), and intravitreal injection of a drug that inhibits the complement system (Zimura). Significant progress is observed in identifying this condition at an early stage, determining clinical features, prognosis, molecular diagnostics and in understanding the pathogenesis of this disease. We are awaiting long-term results of the clinical trials.

KEY WORDS: diagnosis, gene mutations, epidemiology, Stargardt disease, clinical findings, seeking therapies.

\section{INTRODUCTION}

Stargardt disease (STGD) is the most common autosomal recessive childhood and adulthood macular degeneration, caused by mutations in the $A B C A 4$ gene located on chromosome 1 [1]. The ABCA4 gene encodes an ATP-binding cassette membrane protein present in the outer segments of cone and rod photoreceptors which participate in the transport of all-trans-retinal aldehyde.

The consequence of mutation of the ABCA4 gene is the accumulation of a lipofuscin component known as A2E (N-retinylidene-N-retinyl-ethanolamine), toxic for retinal pigment epithelium and photoreceptors [2]. Rarely, STGD can be inherited in an autosomal dominant manner (mutations in ELOVL4 gene on chromosome 6 and PROM1 gene on chromosome 4) $[3,4]$.

Stargardt disease has a prevalence of 1 in 10,000 [5].

\section{CLINICAL FEATURES}

The first symptoms of STGD, such as blurred vision, central scotomas and/or dyschromatopsia, usually are present in childhood or early adolescence, but in some patients, they may be present in later adulthood. At the time of the first ophthal- mological examination, the visual acuity (VA) range is $0.05-1.0$ (Snellen charts). It is worth noting that patients with the onset at a young age have a worse visual prognosis [6]. In the majority of patients $(66 \%)$, in fundus examination, the macula is described as having "beaten bronze metal" appearance with yellowish, "fish-tail" or pisciform lesions [7] (Figure 1).

The maculopathy is progressive and finally the retinal atrophy is detected in the macular area, frequently described as the "bull's eye" pattern (Figure 2).

In the literature, there are available different classifications of Stargardt disease [8-11], which are shown in Table I.

About $2 / 3$ of patients at stage 1 (Fishman classification) remain at this stage for a period of 5 years [12]. About $70 \%$ of patients at stage 2 and $86 \%$ of patients at stage 3 were stable over the follow-up periods of 7.2 and 5.3 years, respectively. At stage 1, the patients have a VA better than 0.1 but the patients at stages 2, 3 and 4 have VA worse than 0.1 .

In patients diagnosed before the age of 20 years, an average VA decline from 0.5 to 0.1 was observed over the followup period of 7 years [7]. In comparison with the young age of diagnosis (before 20 years), the VA decline is less progressive in patients diagnosed between 21-40 and 41-60 years, over 


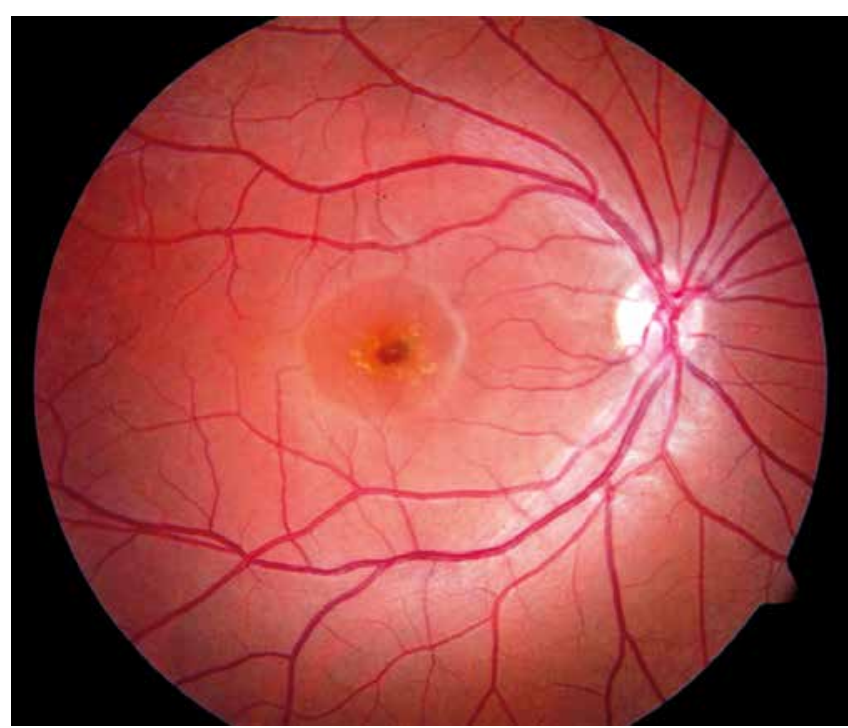

Figure 1. Fundus photo of patient with Stargardt disease. In the fovea, degenerative changes ("beaten bronze"), encircled by flecks are seen

22 and 29 years, respectively. The patients with fovea sparing presented VA of 0.5 or better [7].

Early Stargardt disease (childhood-onset) is connected with severe visual loss, early morphologic changes and often generalized retinal dysfunction. One third of children do not have flecks in fundus examination [9].

\section{DIAGNOSTIC TESTS}

In the diagnosis of Stargardt disease, the following examinations should be performed: anamnesis, routine ophthalmological examination, fundus autofluorescence, spectral-domain optical coherence tomography (SD-OCT), electroretinography, genetic evaluation. Other tests can be useful in diagnosis of Stargardt disease, such as fluorescein angiography, and adaptive optics scanning laser ophthalmoscopy.

The fundus autofluorescence (FAF) imaging method is associated with the autofluorescent properties of lipofuscin. This feature helps in assessment in vivo of lipofuscin distribution in retinal pigment epithelium (RPE) [13]. As STGD is characterized by abnormal level of lipofuscin, FAF may identify early atrophy or flecks or dots before they are clinically evident [14]. An abnormally reduced FAF signal results from the absence or reduction in lipofuscin density and/or $\mathrm{RPE} /$ photoreceptor cell loss, but an abnormally increased FAF signal results from the lipofuscin accumulation which is observed in Stargardt disease. Therefore, FAF helps in diagnosis and estimation of progression of this disease [11] (Table I).

Fundus autofluorescence in different stages of STGD is shown in Figures 3-5.

Spectral-domain OCT provides cross-sectional images of the macula and allows early detection of foveal outer retinal atrophy and loss of the inner ellipsoid band seen in childhood and adulthood onset STGD [15] (Figure 6).

Longitudinally over time, it is possible to analyze the progression of the disease using such parameters as total and

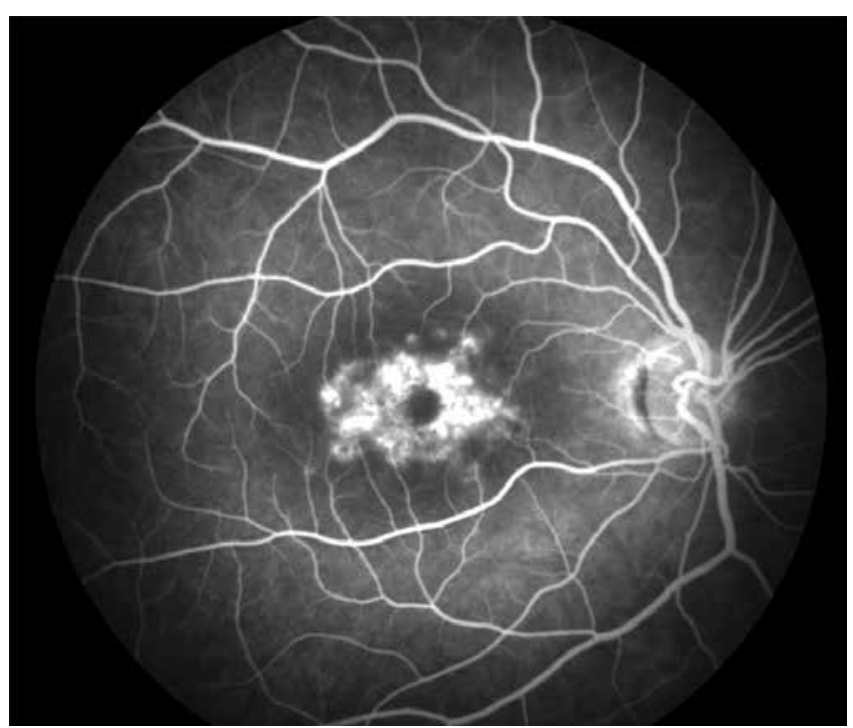

Figure 2. "Bull's eye" pattern of retinal degenerative changes in the macula in a patient with Stargardt disease

outer retinal thickness, macular volume, and inner segment ellipsoid loss. The earliest OCT feature in children is thickening of the external limiting membrane $[15,16]$.

\section{ELECTRORETINOGRAPHY}

Electroretinographic tests, such as multifocal electroretinography (mfERG) $[17,18]$ (Figure 7$)$, pattern ERG (PERG) [19] (Figure 8) and flash full-field ERG (ERG) [10, 11], are helpful in confirming the diagnosis and have prognostic value in STGD. These tests estimate objectively actually generalized bioelectrical function of the retina including the macular region.

In the early stages of STGD, by using the mfERG test it is possible to register foveal dysfunction even in patients with normal fundus appearance or only subtle morphological changes and good VA.

In mfERG examination, the area of dysfunction is usually larger in comparison to other tests' results (static perimetry, OCT, FAF). In the mfERG test, opposite to cone dystrophy, in STGD a significant increase or progressive increase of P1-wave implicit time was not observed.

Pattern ERG is abnormal in almost all patients with STGD, even at the early stages of the disease (reduced amplitudes of P50 and N95 waves, mainly the P50 wave, and implicit time increase of the P50 wave). There is a correlation between the amplitude of PERG and the degree of the photoreceptors' damage seen in the SD-OCT examination.

Based on ERG, the functional loss was described in a classification of three electrophysiological types of STGD (Figure 9).

In all the ERG types of STGD, pattern ERG was absent, suggesting severe macular dysfunction. In type I, ERG was normal, in type II cone ERG was abnormal, but in type III generalized loss of cone and rod function was registered. In the longitudinal study [20] mentioned above, the ERG types of STGD have prognostic implications. The patients with STGD type I had the best prognosis, the patients with STGD 
Table I. Stargardt disease - phenotypic classifications

\begin{tabular}{|c|c|c|c|}
\hline $\begin{array}{l}\text { Fishman [8] } \\
\text { Clinical classification }\end{array}$ & $\begin{array}{l}\text { Fujinami et al. [9] } \\
\text { Early onset - } \\
\text { Fundus Grading System }\end{array}$ & $\begin{array}{l}\text { Lois et al. [10] } \\
\text { ERG }\end{array}$ & $\begin{array}{l}\text { Fujinami et al. [11] } \\
\text { FAF }\end{array}$ \\
\hline $\begin{array}{l}\text { Stage } 1 \\
\text { Macular pigmentary changes or } \\
\text { pisciform ring of flecks within } 1 \mathrm{DD} \text { of } \\
\text { the fovea; EOG, ERG - normal }\end{array}$ & $\begin{array}{c}\text { Grade } 1 \\
\text { Fundus normal }\end{array}$ & $\begin{array}{c}\text { Group } 1 \\
\text { PERG - abnormal } \\
\text { ERG - normal }\end{array}$ & $\begin{array}{l}\text { Type } 1 \\
\text { Low signal at the fovea surrounded by homogeneous } \\
\text { background with or without perifoveal foci of high } \\
\text { or low signal }\end{array}$ \\
\hline $\begin{array}{l}\text { Stage } 2 \\
\text { Flecks extending beyond } 1 D D \text { of the } \\
\text { fovea and nasally to the optic disc; } \\
\text { EOG, ERG - normal }\end{array}$ & $\begin{array}{c}\text { Grade } 2 \\
\text { Macular and/or peripheral } \\
\text { flecks without central atrophy }\end{array}$ & $\begin{array}{c}\text { Group } 2 \\
\text { PERG - abnormal } \\
\text { ERG - cone response } \\
\text { abnormal }\end{array}$ & $\begin{array}{l}\text { Type } 2 \\
\text { Low signal at the macula surrounded by a heterogenous } \\
\text { background and widespread foci of high or low signal } \\
\text { extending anterior to the vascular arcades }\end{array}$ \\
\hline $\begin{array}{l}\text { Stage } 3 \\
\text { Choriocapillaris atrophy of the macula, } \\
\text { diffusely resorbed flecks; EOG, ERG - } \\
\text { subnormal }\end{array}$ & $\begin{array}{c}\text { Grade } 3 \mathrm{~A} \\
\text { Central atrophy without flecks }\end{array}$ & $\begin{array}{c}\text { Group } 3 \\
\text { PERG - abnormal } \\
\text { ERG - cone, rod response } \\
\text { abnormal }\end{array}$ & $\begin{array}{c}\text { Type } 3 \\
\text { Multiple areas of low signal at posterior pole with } \\
\text { heterogenous background and/or foci of high or low } \\
\text { signal }\end{array}$ \\
\hline \multirow{3}{*}{$\begin{array}{l}\text { Stage } 4 \\
\text { Diffusely absorber flecks, } \\
\text { choriocapillaris/RPE atrophy of the } \\
\text { entire fundus; ERG - subnormal }\end{array}$} & $\begin{array}{c}\text { Grade 3B } \\
\text { Central atrophy with macular } \\
\text { and/or peripheral flecks }\end{array}$ & & \\
\hline & $\begin{array}{c}\text { Grade } 3 \mathrm{C} \\
\text { Paracentral atrophy with } \\
\text { macular and/or peripheral } \\
\text { flecks }\end{array}$ & & \\
\hline & $\begin{array}{l}\text { Grade } 4 \\
\text { Multiple extensive atrophy of } \\
\text { the RPE extending beyond the } \\
\text { vascular arcades }\end{array}$ & & \\
\hline
\end{tabular}

FAF - fundus autofluorescence, EOG - electrooculogram, ERG - electroretinogram, PERG - pattern electroretinogram, RPE - retinal pigment epithelium

type II had an intermediate variable prognosis, while the patients with type III had the worst prognosis.

During 10 years of follow-up, $22 \%$ of patients with type I showed transition to type II and type III (11\% in each group). In the case of patients with type II, $47 \%$ of them progressed to type III of STGD.

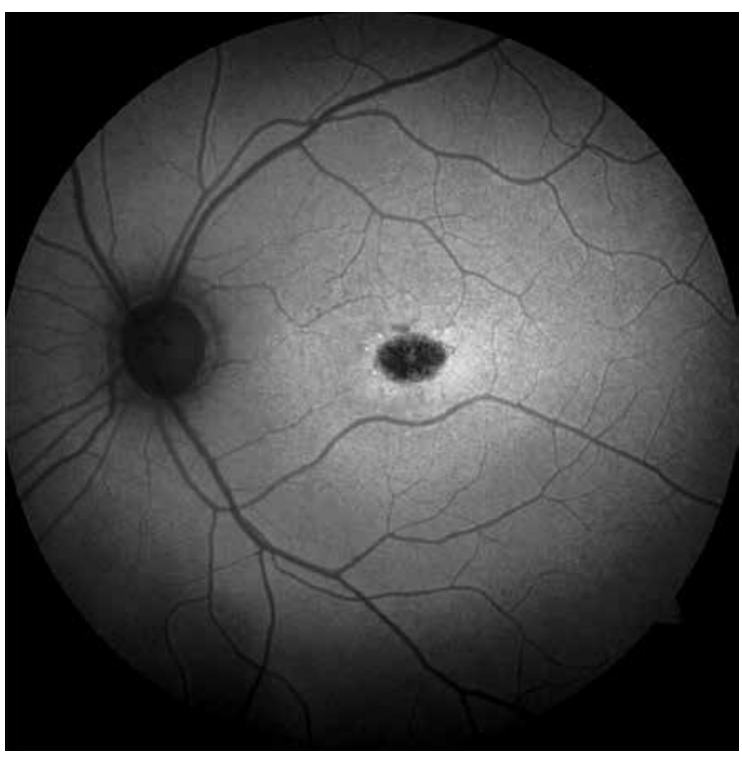

Figure 3. Stargardt disease; fundus autofluorescence (FAF) - stage 1
Clinically significant ERG deterioration was observed in $54 \%$ of all the subjects: only $22 \%$ from type I, $65 \%$ from type II and $100 \%$ from type III. Obtained ERG assists the counseling of the patient in relation to visual prognosis and is also relevant in the patient selection and monitoring of potential therapeutic interventions.

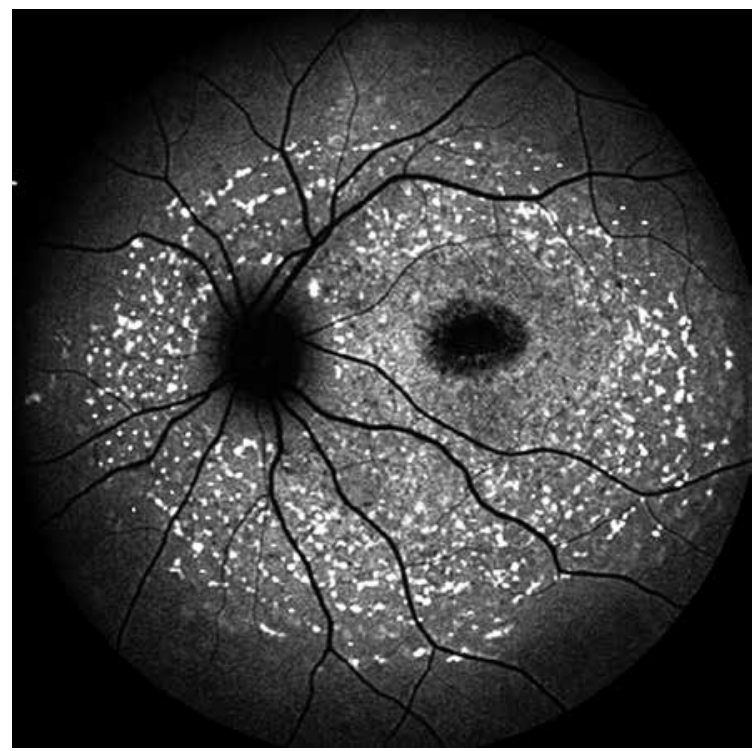

Figure 4. Stargardt disease; fundus autofluorescence (FAF) - stage 2 


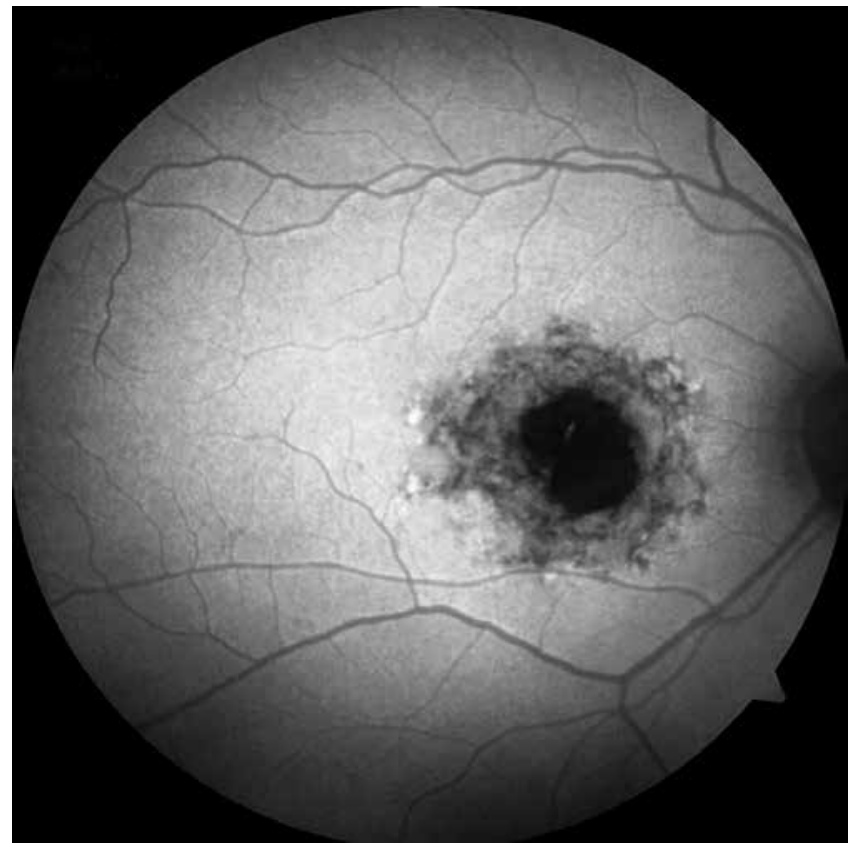

Figure 5. Stargardt disease; fundus autofluorescence (FAF) - early changes in stage 3

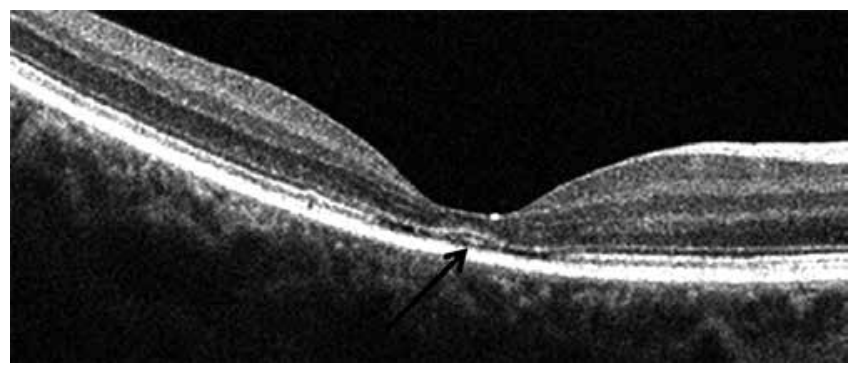

Figure 6. Stargardt disease; spectral domain optical coherence tomography (SD-OCT) in early stage - outer retinal foveal atrophy

\section{ADAPTIVE OPTICS SCANNING LASER OPHTHALMOSCOPY}

Adaptive optics scanning laser ophthalmoscopy (AOSLO) is one of the imaging modalities which allows visualization of the retinal rods and cones [21]. There are two types of AOSLO imaging: confocal and split detector. Confocal im- aging requires an intact photoreceptor outer segment and split-detector imaging requires the presence of intact inner segments. The status of the outer and inner segments of the photoreceptors in STGD may be important in selection of patients for therapeutic interventions.

\section{GENOTYPE-PHENOTYPE CORRELATIONS}

There are more than 900 disease-causing mutations of the $A B C A 4$ gene [22]. That is why STGD is a highly phenotypically heterogenous disease. Notably, the variation in the $A B C A 4$ gene can be associated with cone, cone-rod or rodcone phenotype [23]. In the families with the same mutations, phenotypes may be different, suggesting the influence of other genetic or environmental factors in the pathophysiology of the disease [24]. In general, the missense mutation of the $A B C A 4$ gene is connected with mild and late onset of the disease while nonsense mutations are responsible for more severe early onset disease. The mildest form of STGD (foveal-sparing) is associated with the hypomorphic alleles [25]. It was demonstrated [9] that the patients with $\geq 2$ ABCA4 variants may have early onset and more severe disease, which is very important for counselling for the parents of the affected child.

Generally, STGD is not associated with other ocular or systemic findings.

\section{THERAPEUTIC TRIALS}

There is no proven treatment of Stargardt disease, but early diagnosis is important for low vision interventions, educational and job modifications as well as family planning.

STGD is currently the subject of gene replacement, stem cells and pharmacologic clinical trials.

The aim of gene replacement therapy is to slow or prevent further retinal degeneration. Adeno-associated virus vectors genetically engineered with ABCA4-DNA are injected subretinally.

Currently, there are 2 clinical trials which use an equine infectious anemia virus lentiviral vector. The first (ClinicalTrials.gov identifier: NCT01367444) is a phase I/II clinical trial and demonstrated safety but the efficacy was limited with

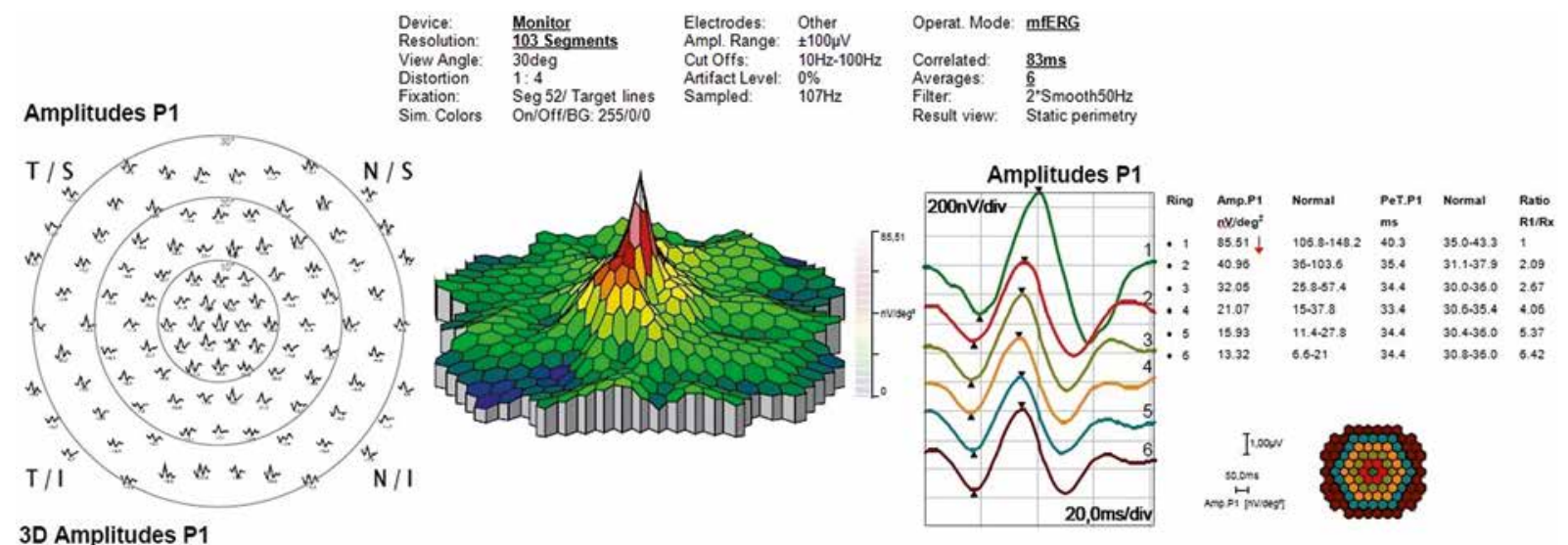

Figure 7. The multifocal electroretinogram (mfERG) recording in a patient at early stage of Stargardt disease. Abnormal function of cone system (reduced response density of $\mathrm{P} 1$ wave in the area covering 5 central degrees is detected; indicated by red arrow) 

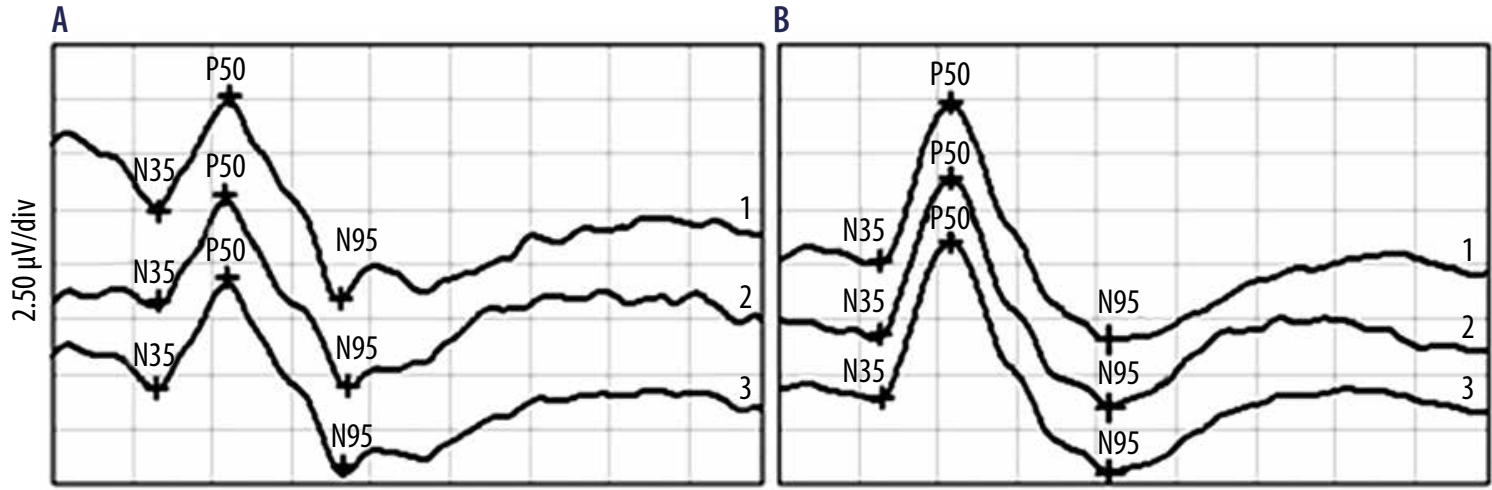

$25.0 \mathrm{~ms} / \mathrm{div}$

$\begin{array}{llr}\text { Channel } & \text { Stimulus } & \text { Ampl., Range. Filter } \\ 1 & \text { Mon. Patt. Rev., } 1^{\circ} \text { Full Field, Ctr.: } 97 \% \text { 2,308 rps, Avg. 200 } 100 \mu V \text { 5-50 Hz } \\ 2 & \text { Mon. Patt. Rev., } 1^{\circ} \text { Full Field, Ctr.: 97\% 2,308 rps, Avg. } 200 \pm 100 \mu V 5-50 \mathrm{~Hz} \\ 3 \text { PERG } & \text { Calculated, Avg.: } 1 & 1 \pm 100 \mu V 5-50 \mathrm{~Hz}\end{array}$

Figure 8. Early stage of Stargardt disease - pattern electroretinogram (PERG) recording with reduced amplitude of P50 wave (A) in comparison with normal PERG from normal subject (B)

patients with severe disease [26]. The second trial (ClinicalTrials.gov identifier: NCT01736592) is assessing the longterm safety and tolerability with patients from the first clinical trial without further interventions for a period of 15 years. For gene therapy of STGD, a promising alternative to the viral vector can be nanoparticle technology, which was proven in a mouse model [27].
Retinal pigment epithelium cells have a significant role in the pathogenesis of STGD. Stem cell therapy is an option to rejuvenate or replace damaged RPE cells in this disease. Stem cells can be derived from human embryonic, induced pluripotent or adult stem cells. RPE cells from human embryonic stem cells (hESC) were injected in the subretinal area in mice with retinal degeneration and the cells sustained the visual

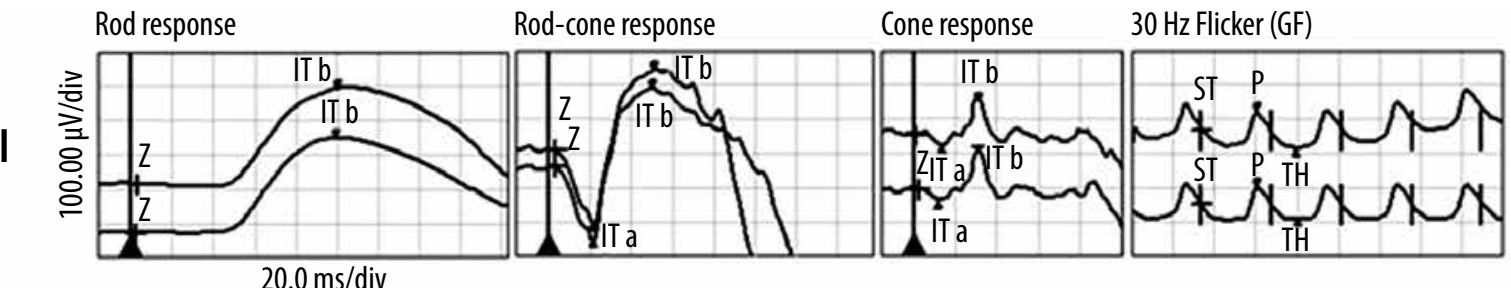

Rod response $20.0 \mathrm{~ms} / \mathrm{div} \quad$ Rod-cone response

Cone response

$30 \mathrm{~Hz}$ Flicker (GF)

II
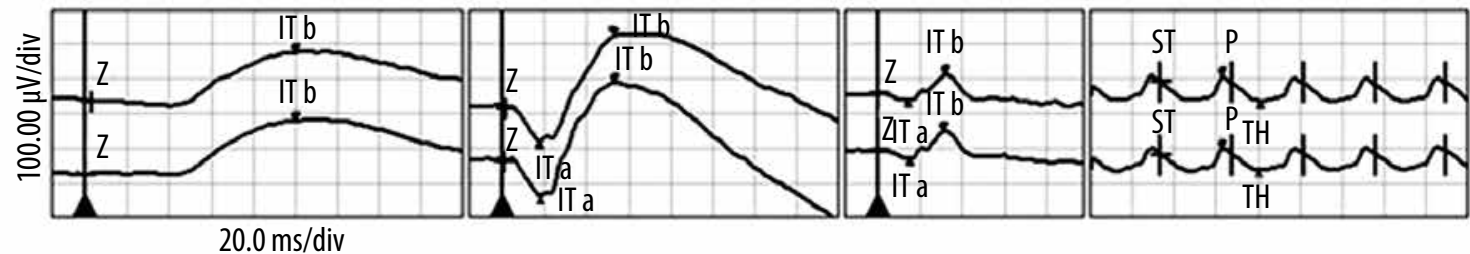

Rod response

III

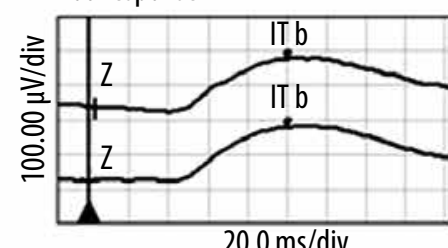

Rod-cone response

Cone response

$30 \mathrm{~Hz}$ Flicker (GF)
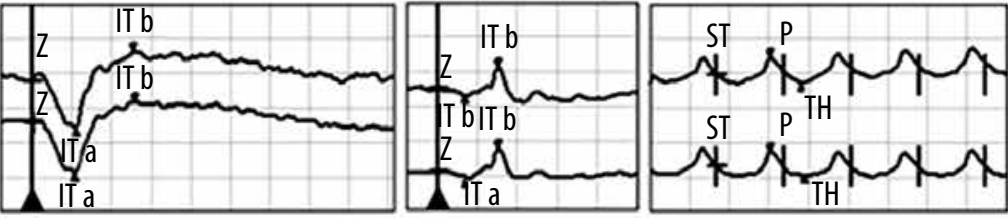

Rod response

Rod-cone response

Cone response
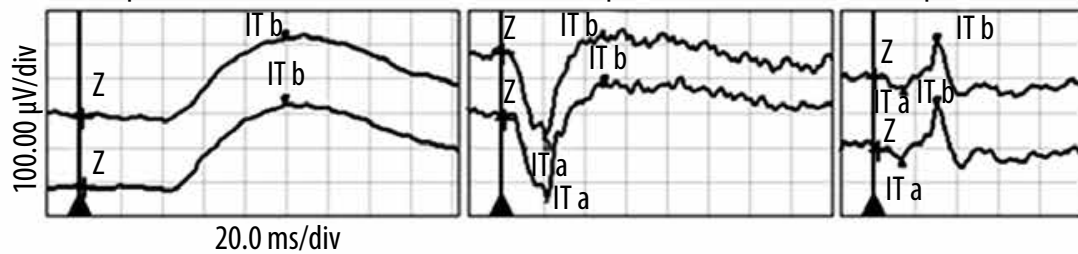

$30 \mathrm{~Hz}$ Flicker (GF)

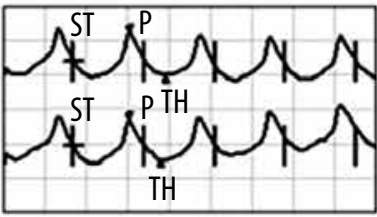

Figure 9. Three electrophysiological types of Stargardt disease. Type I - normal electroretinogram (ERG), type II - abnormal cone ERG (reduced photopic amplitude of b-wave), type III - abnormal rod and cone ERG (reduced scotopic and photopic amplitude of b-wave), $\mathrm{N}$ - normal ERG from healthy subject 
function and photoreceptor integrity [28]. Currently, a phase $1 / 2$ clinical trial is under evaluation of efficacy of subretinal transplantation of the hESC-derived RPE cells in patients with STGD (NCT01345006). In this trial, no signs of acute rejection were observed and the 3-year follow-up suggested safety [29]. Further studies of efficacy are ongoing.

Pharmacological, investigational therapies in clinical trials include visual cycle modulators reducing accumulation of vitamin A dimers and complement inhibitors.

The $A B C A 4$ gene acts as a membrane transporter for the recycling of chromophores according to the visual cycle. The mutations of this gene are the cause of the abnormalities of the membrane transport of chromophores leading to accumulation of vitamin A byproducts or dimers (bisretinoids) in the RPE cells. It is known that vitamin A dimers are toxic to RPE cells and play a significant role in lipofuscin formation in STGD [30]. Study results in mice suggested that slowing the intrinsic reactivity of vitamin A to dimerize slows lipofuscin accumulation in RPE cells and finally could slow deterioration of retinal cells in STGD [31]. A phase1 trial (NCT02230228), assessing safety of an oral dose of C20-D3-vitamin A molecule ALK-001 (Alkeus) once a day which impedes vitamin A dimerization, was finished with success. Currently, a phase 2 (TEAS study) trial (NCT02402660) is being carried out in 50 patients with termination planned in the year 2021.

The visual cycle can be modulated by Emixustat HCI (ACU-4429, Acucela) - a nonretinoid derivative of retinylamine. This drug delivered per os reduces the conversion of all-trans-retinylesters to 11-cis-retinol and prevents accumulation of A2E and has potential STGD treatment value. Data from a phase 1 study of Acucella suggested that this drug was well tolerated [32]. We are waiting for the results of a phase
3 multicenter, randomized, double-masked, placebo-controlled study (NCT03772665) of 162 patients with STGD.

In RPE cells, A2E and other bisretinoids may be the cause of inflammation by activating the complement system [33]. Avacincaptad pegol (Zimura, Iveric Bio) is a C5 complement inhibitor which may prevent formation of the membrane attack complex and reduce destruction of the cell membranes and finally the death of cells. A phase $2 \mathrm{~b}$ double masked sham controlled study (NCT 03364153) with Zimura delivered by intravitreal injection included 95 STGD patients. The primary endpoint is the rate of change of the area of the ellipsoid zone measured by SD-OCT.

In conclusion, STGD is the most commonly inherited cause of visual loss in childhood and adulthood. This maculopathy is a highly phenotypically and genotypically heterogeneous disease. Significant progress is observed in identifying this condition at an early stage, determining clinical features, prognosis, molecular diagnostics and in understanding the pathogenesis of the disease. In a patient with suspected STGD it is necessary to perform the following tests: routine ophthalmological examination, FAF, SD-OCT, ERG, mfERG or PERG. For confirmation of the clinically suggested diagnosis, genetic evaluation is necessary. Long-term prospective studies of the natural course of the disease, and analysis of genotypicphenotypic and structural-functional relationships, are important for the prognostic evaluation and genetic counseling as well as for a more appropriate selection of patients to be included in the clinical trials.

\section{DISCLOSURE}

The authors declare no conflict of interest.

\section{References}

1. Allikmets R, Singh N, Sun H, et al. A photoreceptor cel-specific ATP-binding transporter gene (ABCR) is mutated in recessive Stargardt macular dystrophy. Nat Genet 1997; 15: 236-246.

2. Sparrow JR, Gregory-Roberts E, Yamamoto K, et al. The bisretinoids of retinal pigment epithelium. Prog Retin Eye Res 2012; 31: 121-135.

3. Agbaga MP, Tam BM, Wong JS, et al. Mutant ELOVL4 that causes autosomal dominant stargardt-3 macular dystrophy is misrouted to rod outer segment disks. Invest Ophthalmol Vis Sci 2014; 55: 3669-3680.

4. Kniazeva M, Chiang MF, Morgan B, et al. A new locus for autosomal dominant Stargardt-like disease maps to chromosome 4. Am J Hum Genet 1999,64: 1394-1399.

5. Michaelides M, Hunt DM, Morre AT. The genetics of inherited macular dystrophies. J Med Genet 2003; 40: 641-650.

6. MiraldiUtz V, Coussa RG, Marino MJ, et al. Predictors of visual acuity and genotype-phenotype correlates in a cohort of patients with Stargardt disease. Br J Ophthalmol 2014; 98: 513-518.

7. Rotenstreich Y, Fishman GA, Anderson RJ. Visual acuity loss and clinical observations in a large series of patients with Stargardt disease. Ophthalmology 2003; 110: 1151-1158.

8. Fishman GA. Fundus flavimaculatus. A clinical classification. Arch Ophthalmol 1976; 94: 2061-2067.

9. Fujinami K, Zernant J, Chana RK, et al. Clinical and molecular characteristics of childhood-onset Stargradt disease. Ophthalmology 2015; 122: 326-334.

10. Lois N, Holder GE, Bunce C, et al. Phenotypic subtypes of Stargardt macular dystrophy-fundus flavimaculatus. Arch Ophthalmol 2001; 119: 359-369.

11. Fujinami K, Lois N, Mukherjee R, et al. Alongitudinal study of Stargardt disease: quantative assesment of fundus autofluorescence, progression, and genotype correlations. Invest Ophthalmol Vis Sci 2013; 54: 8181-8190.

12. Kim LS, Fishman GA. Comparison of visual acuity loss in patients with different stages of Stargardt's disease. Ophthalmology 2006; 113: 1748-1751.

13. Delori FC, Dorey CK, Staurenghi G, et al. In vivo fluorescence of the ocular fundus exibits retinal pigment epithelium lipofuscin characteristics. Invest Ophthalmol Vis Sci 1995; 36: 718-729.

14. Boon CJ, Jeroen Klevering B, Keunen JE, et al. Fundus autofluorescence imaging in retinal dystrophies. Vision Re 2008; 48: 2569-2577. 
15. Strauss RW, Munoz B, Wolfson Y, et al. Assessment of estimated retinal atrophy progression in Stargardt macular dystrophy using spectra-domain optical coherence tomography. Br J Ophthalmol 2016; 100: 956-962.

16. Lee W, Nõupuu K, OII M, et al. The external limiting membranę in early - onset Stargradt disease. Invest Ophthalmol Vis Sci 2014; 55: 6139-6149.

17. Kuniyoshi K, Terasaki H, Arai M, Hirose T. Multifocal electroretinograms in Stargardt's disease/fundus flavimaculatus. Ophthalmologica 2014; 232: 118-125.

18. Shechtman D, Woods A, Pizzimenti J. Use of multifocal ERG and OCT for diagnosing Stargardt's disease. Clin Exp Optom 2011; 94: 309-313.

19. Lenassi E, Jarc-Vidmar M, Glavac D, Hawlina M. Pattern electroretinography of larger stimulus field size and spectral-domain optical coherence tomography in patients with Stargardt disease. Br J Ophthalmol 2009; 93: 1600-1605.

20. Fujinami K, Lois N, Davidson AE, et al. Longitudinal study of stargardt disease: clinical and electrophysiologic assessment, progression, and genotype correlations. Am J Ophthalmol 2013; 155: 1075-1088.

21. Scoles D, Sulai YN, Langlo CS, et al. In vivo imaging of human cone photoreceptor inner segments. Invest Ophthalmol Vis Sci 2014; 55: 4244-4251.

22. Zernant J, Xie YA, Ayusso C, et al. Analysis of the ABCA4 genomic locus in Stargradt disease. Hum Mol Genet 2014; 23: 6797-6806.

23. Klevering BJ, Deutman AF, Maugeri A, et al. The spectrum of retinal phenotypes caused by mutations in the ABCA4 gene. Graefes Arch Clin Exp Ophthalmol 2005; 243: 90-100.

24. Burke TR, Tsang SH, Zernant J, et al. Familial discordance in Stargardt disease. Mol Vis 2012; 18: 227-233.

25. Zernant J, Lee W, Collison FT, et al. Frequent hypomorphic alleles account for a significant fraction of ABCA4 disease and distinguish it from age-related macular degeneration. J Med Genet 2017; 54: 404-412.

26. Smith J, Ward D, Michaelides $M$, et al. New and emerging technologies for the treatment of inherited retinal diseases: a horison scanning review. Eye 2015; 29: 1131-1140.

27. Han Z, Conley SM, Makkia RS, et al. DNA nanoparticle-mediated ABCA4 delivery rescues Stargardt dystrophy in mice. J Clin Invest 2012; 122: 3221-3226.

28. Lu B, Malcuit C, Wang S, et al. Long term-safety and function of RPE from human embrionic stem, cells in preclinical models of macular degeneration. Stem Cells 2009; 27:2126-2135.

29. Schwartz SS, Regillo C, Lam BL, et al. Human embryonic stem cell-derived retinal pigment epithelium in patients with age-related macular degeneration and Stargardt's macular dystrophy: follow-up of two open label phase 1/2 studies. Lancet 2015; 385: 509-516.

30. Sparrow JR, Fishkin N, Zhou J, et al. A2E, a byproduct of the visual cycle. Vision Res 2003; 43: 2983-2990.

31. Charbel IP, Barnard AR, Herrmann P, et al. Rescue of the Stargardt phenotype in ABCA4 knockout mice through inhibition of vitamin A dimerization. Proc Natl acad Sci USA 2015; 112: 8415-8420.

32. Kubota R, Boman NL, David R, et al. Safety and effect on rod function of ACU-4429, a novel small-molecule visual cycle modulator. Retina 2012; 32: 183-188.

33. Zhou J, Kim SR, Westlund BS, et al. Complement activation by bisretinoid constituents of RPE lipofuscin. Invest Ophthalmol Vis Sci 2009; 50: 1392-1399. 